\title{
New Methods for Predicting Productivity and Critical Production Rate of Horizontal Wells
}

\author{
Hong'en Dou*
}

RIPED, PetroChina, P.R. China

\begin{abstract}
Horizontal well technology is still applied widely in the world. Some researchers and oilfield operators of horizontal wells are still using improper productivity predicting equations and critical production rate calculations. Therefore, this paper analyzes some productivity forecast equations and critical coning rate calculation procedures for horizontal wells focusing on Joshi's equation of predicting productivity and Chaperon's equation of calculating critical coning rate used in reservoir engineering. This paper suggests that effective permeability should be used in calculating horizontal well productivity and points out an error existing in Joshi's equation. It also evaluates and introduces several equations for critical production rate. Furthermore, this paper derives two new equations for predicting horizontal well productivity and a general predictive equation for horizontal well critical coning rate, applying the Mirror Effect Theory. Field applications show that the relative error between generally accepted methods of prediction are less than $17 \%$ and the new calculation method is more accurate than that of Joshi's equation. Moreover, using actual oilfield examples, the modified Chaperon's equation was verified.
\end{abstract}

\section{INTRODUCTION}

Since 1986, the number of horizontal wells has grown from 30 to tens of thousands, and the prediction of horizontal well productivity has become very important. At the beginning of 1980 s $[1,2]$ derived some equations to predict horizontal well productivity. In 1986 and 1991 Joshi [3, 4] rederived an equation by using the complex potential method. A term of $\ln \left[\mathrm{h} /\left(2 \mathrm{r}_{\mathrm{w}}\right)\right]$ in the nominator of the Joshi equation is different from that of $\ln \left[\mathrm{h} /\left(2 \pi \mathrm{r}_{\mathrm{w}}\right)\right]$ in the Renard equation. The difference is only $\pi$ in Renard equation, but no difference had been proved in the accuracy of the results by the author of this paper.

Joshi assumed that the fluid flowing through multiporous media could be divided into two components of vertical and horizontal flow. In fact, these two parts are mutually perpendicular and mutually contained. But in the Joshi equation, this influence was ignored, and therefore its prediction results were not quite accurate.

In the paper, the Joshi equation was corrected by using average effective permeability, error between oilfield actual value and the calculated value of the corrected equation reduce to $58 \%$. Also, a new equation was derived by the mirror image theory $[6,7]$ to precisely predict the productivity of a horizontal well in a homogeneous formation.

For water coning/cresting of bottom water reservoirs developed with horizontal wells, the main concern is the reduction of production. Control of water coning/cresting is a big technological problem that has been faced by petroleum engineers for a long time. Many researchers have spent a great deal of energy in studying the calculation method for critical

*Address correspondence to this author at the RIPED, PetroChina, P.R. China; E-mail: douhongen@petrochina.com.cn or dohe@public.bta.net.cn production rate $[1,5,8-12]$ in horizontal wells for water coning/cresting. However, based on the same production well data, different equations will give different results, which can cause great trouble to the petroleum engineers. In the paper, a new calculation formula for critical production was introduced.

\section{DISCUSSION OF PRODUCTIVITY EQUATIONS}

In 1986 and 1991 Joshi $[3,4]$ has given several productivity equations as follows:

Joshi equation

$Q_{h}=\frac{2 \pi K_{h} h}{\mu B_{o}} \frac{\Delta P}{\ln \frac{a+\sqrt{a^{2}-(L / 2)^{2}}}{L / 2}+\frac{h}{L} \ln \frac{h}{2 r_{w}}}$

where $a=0.5\left(0.5+\sqrt{0.25+\left(1 /\left(L / 2 r_{w}\right)\right)^{4}}\right)^{0.5}$

Borison equation

$Q_{h}=\frac{2 \pi K_{h} h}{\mu B_{o}} \frac{\Delta P}{\ln \frac{4 r_{e h}}{L}+\frac{h}{L} \ln \frac{h}{2 \pi r_{w}}}$

Giger equation

$Q_{h}=\frac{2 \pi K_{h} h}{\mu B_{o}} \frac{\Delta P}{\ln \frac{1+\sqrt{1-\left(L / 2 r_{e h}\right)^{2}}}{L / 2 r_{e h}}+\frac{h}{L} \ln \frac{h}{2 \pi r_{w}}}$

Renard and Dupuy equation

$Q_{h}=\frac{2 \pi K_{h} h}{\mu B_{o}} \frac{\Delta P}{\ln \frac{1+\sqrt{1-(L / 2 a)^{2}}}{L / 2 a}+\frac{h}{L} \ln \frac{h}{2 \pi r_{w}}}$

It is obvious that the difference between the Joshi equation and the Renard and Dupuy equation is a constant term 
$\pi$ in the nominator of the Joshi equation. In addition, the only difference between the equations of Giger and Joshi is Giger assumed $a=r_{e h}$. But the Borison equation thought $a=r_{e h}$, and when value $L / 2 r_{e h}$ is very little, and it can be ignored, the Renard and Dupuy equations were turned into the Borison equation; here, what must be mentioned, the equation (1) to (4) can be suitably applied to isotropic reservoir formation. However, when the above equations are changed into reservoir engineering units, $2 \pi$ can be replaced by $5.4259 \times 10^{2}$.

\section{DISCUSSION OF THE JOSHI EQUATION}

Joshi (1986) [3] assumed that fluid flowing into a horizontal well may be divided into two parts, one is flowing on a horizontal plane and the other is flowing on the vertical plane and come to the ellipse drainage in the horizontal plane and cylindrical drainage in vertical plane. The flow resistance in the two planes was calculated respectively, using a productivity formula similar in principle with $\mathrm{Ohm}$ and Darcy. It is clear that the Joshi equation should not eliminate this overlapping factor and thus result in error in the productivity calculation of horizontal wells.

Using average effective permeability, the productivity prediction of anisotropy formation is reasonable, and people still divide permeability into the horizontal and the vertical. In fact, fluids flowing through porous media in the vertical and the horizontal planes influence and interact on each other in the reservoir. The permeability direction in formation will be disordered and unsystematic because the actual effect in the reservoir will not be really simulated. The permeability value is different in the different directions; therefore, fluid will flow toward the direction that has the low flow resistance. The three permeability of $\mathrm{K}_{\mathrm{x}}, \mathrm{K}_{\mathrm{y}}$ and $\mathrm{K}_{\mathrm{z}}$ were difficult to acquire. Moreover, by using Muskat's $Z=h \sqrt{K_{h} / K_{v}}$ the horizontal well production equation of the isotropic formation was transformed into anisotropic formation as follows:

$Q_{h}=\frac{2 \pi K_{h} h}{\mu B_{o}} \frac{\Delta P}{\ln \frac{a+\sqrt{a^{2}-(L / 2)^{2}}}{L / 2}+\frac{\beta h}{L} \ln \frac{\beta h}{2 r_{w}}}$

where $\beta=\sqrt{K_{h} / K_{v}}$

It is clear that in equation (5), production rate $Q_{h}$ is inversely proportional to $\beta$, and is directly proportional to $K_{h}$, but $\beta$ is associated with $K_{h}$ and $K_{v}$. Only when the $\beta$ value is very low $(0<\beta<1)$, the $Q_{h}$ value can increase, however, $Q_{h}$ change is also considerably sensitive to $K_{h}$. For equation (5), when $K_{h}$ is less than $K_{v}$ and $K_{h}$ is much lower, the $Q_{h}$ value is also lower. In the case, the actual value of $Q_{h}$ from the oilfield is more than the calculation value. By contrast, when $K_{h}$ is more than $K_{v}$, and $K_{h}$ is much bigger, the $Q_{h}$ value is also bigger; however, the actual value of $Q_{h}$ from oilfield is not as high as that. In general, when we calculate $Q_{h}$, no matter what $K_{h}$ and $K_{v}$ are, neither is stable. The paper suggests replacing the horizontal permeability or vertical permeability with average effective permeability, as follows:
$K=\sqrt{K_{h} K_{v}}$

Horizontal well productivity calculation applies the equation (6) is reasonable. Substituting it into equation (5) we have a corrected Joshi equation:

$Q_{h}=\frac{2 \pi K h}{\mu B_{o}} \frac{\Delta P}{\ln \frac{a+\sqrt{a^{2}-(L / 2)^{2}}}{L / 2}+\frac{h}{L} \ln \frac{h}{2 r_{w}}}$

\section{NEW EQUATION OF PRODUCTIVITY PREDICTION IN HORIZONTAL WELL}

We assume the horizontal well is the same as a straight vertical well; the horizontal length is equal to the reservoir thickness. Furthermore, we assume the producer in the infinite formation, and a new productivity equation was derived by the Mirror Effect Principle [6,7].

According to the complex potential principle [6], we obtain

$\Phi=\frac{q}{2 \pi} \sum_{+\infty}^{+\infty} \ln r+c$

where:

$$
\begin{aligned}
& \Phi=\frac{q}{2 \pi} \sum_{+\infty}^{+\infty}\left[\ln \sqrt{X^{2}+\left(Z-2 n h-Z_{w}\right)^{2}}+\right. \\
& \left.\ln \sqrt{X^{2}-\left(Z-2 n h+Z_{w}\right)^{2}}\right]+c \\
& \Phi=\frac{q}{4 \pi} \ln \left(\operatorname{ch} \frac{\pi X}{h}-\cos \frac{\pi\left(Z-Z_{w}\right)}{h},\right)\left(\operatorname{ch} \frac{\pi X}{h}-\cos \frac{\pi\left(Z+Z_{w}\right)}{h}\right)+c
\end{aligned}
$$

Through simplification (see Appendix A):

$$
\Delta \Phi=\frac{q}{2 \pi}\left(\frac{\pi r_{e}}{h}+\ln \frac{h}{2 \pi r_{w}} \sin \frac{\pi Z_{w}}{h}\right)
$$

When a horizontal well was drilled in the reservoir center without eccentricity, $Z_{w}=h / 2$, the equation (11) was reduced into:

$\Delta \Phi=\frac{q}{2 \pi}\left(\frac{\pi r_{e}}{h}+\ln \frac{h}{2 \pi r_{w}}\right)$

Therefore:

$\Delta \Phi=\frac{K}{\mu} \Delta P$

Combining equation (12) and (13), we obtain:

$q=\frac{2 \pi K}{\mu}\left(\frac{\Delta P}{\frac{\pi r_{e}}{h}+\ln \frac{h}{2 \pi r_{w}}}\right)$

For a horizontal well of length $\mathrm{L}$, production rate $\mathrm{Q}$ is calculated as:

$$
Q=q L=\frac{2 \pi K}{\mu} \frac{\Delta P}{\frac{\pi r_{e}}{L}+\frac{h}{L} \ln \frac{h}{2 \pi r_{w}}}
$$

Equation (15) represents horizontal well production rate in infinite formation: 
Table 1. Reservoir Basic Parameters

\begin{tabular}{|c|c|c|c|c|c|c|c|c|c|c|c|}
\hline Well No. & $L(m)$ & $h(m)$ & $r_{w}(m)$ & $r_{e}(m)$ & $\mu\left(\boldsymbol{m} \boldsymbol{P}_{a} \cdot \mathbf{s}\right)$ & $\boldsymbol{\beta}$ & $\boldsymbol{K}_{\boldsymbol{h}}\left(\times 10^{-3} \mu m^{2}\right)$ & $\boldsymbol{K}_{\boldsymbol{v}}\left(\times 10^{-3} \mu m^{2}\right)$ & $P_{e}\left(M P_{a}\right)$ & $\boldsymbol{P}_{w}\left(\boldsymbol{M P}_{a}\right)$ & $\boldsymbol{B}_{o}$ \\
\hline L-3 & 368 & 36.5 & 0.12 & 100 & 41 & 1.00 & 10.0 & 7.50 & 17.33 & 4.0 & 1.00 \\
\hline S-1 & 309 & 14.0 & 0.061 & 239.4 & 3.0 & 1.13 & 1.30 & 0.04 & 17.0 & 4.0 & 1.13 \\
\hline R-1 & 300 & 6.5 & 0.1094 & 170 & 6.2 & 1.08 & 9.50 & 3.00 & 19.5 & 19.2 & 1.083 \\
\hline
\end{tabular}

$Q=\frac{2 \pi K h}{\mu B_{o}} \frac{\Delta P}{\frac{\pi r_{e}}{L}+\frac{h}{L} \ln \frac{h}{2 \pi r_{w}}}$

To transform the above equation into actual reservoir engineering unit (S.I system) equation (16) is expressed as:

$Q=\frac{5.4295 \times 10^{2} K h}{\mu B_{o}} \frac{\Delta P}{\frac{\pi r_{e}}{L}+\frac{h}{L} \ln \frac{h}{2 \pi r_{w}}}$

\section{COMPARISON OF NEW EQUATION AND THE JOSHI EQUATION}

Three horizontal wells, as exampled in China, are Lengping 3 (L-3) in Liaohe oilfield, Shuping 1 (S-1) in Daqing oilfield and Renping 1 (R-1) in Huabei I oilfield. The base data is shown in Table $\mathbf{1}$.

The procedures for estimating these three wells are:

1. Calculate horizontal well production rate using equation (17).

2. Estimate well production rate using Joshi's equation (5).

3. Estimate well production rate using corrected Joshi's equation (7).

4. Compare results of equation (17), (5) and (7).

Actual reservoir data of the three oilfields are shown in Table 1; several estimated results for horizontal well productivity are listed in Table 2 .

\section{DISCUSSION ON THE FORMULATION OF THE CRITICAL PRODUCTION RATE OF HORIZONTAL WELLS}

\section{Definition of Critical Production Rate}

The concept of critical production rate has always been used in vertical well production. This concept is expressed as: "the critical production rate is the maximum oil production rate without water or gas" $[3,4]$. Although Joshi had introduced it in the horizontal wells, in practice, many scholars thought that the critical production rate is production rate of the first water drop to appear on the surface in a bottom water reservoir. Actually, water had broken through the water-oil contact (WOC) before the first water drop appears on the surface, so this production rate cannot be defined as critical production. It has been considered [11] that the definition of critical production rate in a bottom water reservoir may be defined as the production rate at which the plane of WOC about starts to deform. The author of the paper thinks that the definition accords with the actual reservoir development.

\section{Discussion and Correction of Chaperon Formulation}

Chaperon's formulation (1986) [8] was derived by using the Laplace equation under the postulation of constant pressure boundary. The author found some errors in the Chaperon derivation, which will be discussed in detail in the following.

Chaperon Derivation was expressed as

$Q_{c}=3.486 \times 10^{-5}\left(\frac{\Delta \rho K h L}{\mu}\right) \times 2 \frac{1-\cos \left(\frac{\pi Z_{s c}}{h}\right)}{\sin \frac{\pi Z_{s c}}{h}}$

But Chaperon recommended using

$Q_{c}=3.486 \times 10^{-5}(\Delta \rho h) \frac{K_{h} h L F}{X_{A} \mu}$

In the equation, when the value of $\mathrm{F}$ is 4 , which is given by Chaperon, the greatest calculation error is only $44 \%$. Later in 1986, Joshi [3] has regressed F, and given a formula as:

$$
\begin{aligned}
& F=3.9624955+0.0616348 \alpha-0.000548 \alpha^{2} \\
& \alpha=\left(\frac{X_{A}}{h}\right) \sqrt{K_{y} / K_{h}}
\end{aligned}
$$

Below will discuss Chaperon's formula error and make a modification to the Chaperon equation.

From the trigonometric function, the following expression can be obtained:

$$
\operatorname{tg} \frac{\pi Z_{s c}}{2 h}=\frac{1-\cos \left(\frac{\pi Z_{s c}}{h}\right)}{\sin \left(\pi Z_{s c} / h\right)}
$$

\begin{tabular}{|c|c|c|c|c|c|c|c|}
\hline Well No. & $Q_{a}(t / d)$ & $Q_{n}(t / d)$ & $Q_{n e}(\%)$ & $Q_{j}(t / d)$ & $Q_{j e}(\%)$ & $Q_{j c}(t / d)$ & $Q_{j c e}(\%)$ \\
\hline L-3 & 44.3 & 43.87 & 0.97 & 98.33 & 121.96 & 36.18 & 18.3 \\
\hline S-1 & 8.88 & 7.33 & 17.5 & 21.24 & 139.3 & 14.05 & 58.2 \\
\hline R-1 & 89.9 & 89.66 & 0.29 & 16.00 & 82.20 & 119.75 & 33.2 \\
\hline
\end{tabular}

Table 2. Comparison of the Calculation Value with Oilfield Actual Value 
Then, equation (1) can also be expressed as:

$Q_{c}=3.486 \times 10^{-5} \frac{\Delta \rho K h L}{\mu} 2 \operatorname{tg} \frac{\pi Z_{s c}}{2 h}$

When $Z_{s c}$ is approaching zero, $Q_{c}$ will approach zero; $Z_{s c}$ approaches $\mathrm{h}, \mathrm{Q}_{\mathrm{c}}$ will be infinite. From the above discussion we can see that the critical production will be infinite when the coning height is $h$. Obviously, this is impossible.

The error is caused by the use of a dimensionless critical production rate equation:

$q^{*}=2 \frac{1-\sin \left(\pi Z_{s c} / h\right)}{\cos \pi Z_{s c} / h}$

In 1990, Dikken [12] had shown the error of Chaperon's equation, but there is no detailed derivation in his paper.

By using the Chaperon equation, the calculated critical rate is bigger than that from other equations. This basic equation was given by Chaperon:

$\Delta P=\frac{Q \mu}{2 \pi L K} \ln \left(\operatorname{ch} \frac{\pi X_{A}}{h}-\cos \frac{\pi Z_{w}}{h}\right)$

Bottom water reservoir does not happen to coning/cresting under condition:

$\Delta P \leq \Delta \rho g\left(h-Z_{w}\right)$

The following equation can be derived from equation (24) and (25)

$Q_{c v}=5.317 \frac{K h}{\mu B_{o}} \frac{\Delta \rho h\left(1-Z_{w} / h\right)}{\frac{h}{L} \ln \left(\operatorname{ch} \frac{\pi X_{A}}{h}-\cos \frac{\pi Z_{w}}{h}\right)}$

Now, let us discuss the correction to the formula. When $h$ approaches $Z_{w}$, the production is zero. This is right because of water breakthrough into wellbore.

\section{Example and Discussion}

Let us take the No. 3 horizontal well in Tazhong of Tarim oilfield in China (the well is TZ4-27-H14), as an example, the reservoir data of this well are listed in Table 3.

Using data in Table 1, the following represents the results from equation (19) and (26) respectively: $Q_{c}=512.99 \mathrm{~m}^{3} / \mathrm{d}$ and $Q_{c v}=143.05 \mathrm{~m}^{3} / \mathrm{d}$. The critical production calculation value of the Chaperon equation is 3.58 times larger than the corrected equation value. The reason leading to this error is that Chaperon and Joshi did not determine the critical height and assumed it only by experience value. Unfortunately, this experience value is quite deviation from the actual results.

\section{Giger's Formula}

In 1986, IFP (Institut Francais du Petrole), Giger (1986) [9] derived a formula to calculate the critical production of horizontal wells with bottom water coning/cresting, on the basis of the postulation, the three sides are closed and impermeable. It is expressed as follows:

$Q_{c}=1.251 \times 10^{-3} \frac{\Delta \rho K_{h} X_{A}}{\mu B_{o}}\left[\left(1+\frac{16}{3}\left(\frac{h}{X_{A}}\right)^{2}\right)^{1 / 2}-1\right] L$
The result was obtained from equation (27) using the data in Table 3 .

$Q_{c}=785 \mathrm{~m}^{3} / \mathrm{d}$

Table 3. Reservoir Parameters of Well TZ4-27-H14

\begin{tabular}{|c|c|}
\hline Surface oil density $\rho_{s}$ & $0.84 \mathrm{~g} / \mathrm{cm}^{3}$ \\
\hline Underground water density $\rho_{w}$ & $1.06 \mathrm{~g} / \mathrm{cm}^{3}$ \\
\hline Underground oil density $\rho_{o}$ & $0.65 \mathrm{~g} / \mathrm{cm}^{3}$ \\
\hline Oil volume factor $B_{o}$ & $1.615 \mathrm{~m}^{3} / \mathrm{m}^{3}$ \\
\hline Producing pressure drop $\Delta P$ & $1.26 \mathrm{MP}_{\mathrm{a}}$ \\
\hline Viscosity of underground oil $\mu$ & $0.29 \mathrm{~m} \mathrm{P_{ \textrm {a } } \mathrm { s }}$ \\
\hline Horizontal permeability $K_{h}$ & $0.164 \mu \mathrm{m}^{2}$ \\
\hline Vertical permeability $K_{v}$ & $0.0492 \mu \mathrm{m}^{2}$ \\
\hline Diameter of wellbore $r_{w}$ & $0.11 \mathrm{~m}$ \\
\hline Diameter of drainage $r_{e}$ & $300 \mathrm{~m}$ \\
\hline Thickness of reservoir $h$ & $33.5 \mathrm{~m}$ \\
\hline Length of horizontal section $L$ & $444.4 \mathrm{~m}$ \\
\hline Distance from bottom of oil layer $Z_{w}$ & $22.75 \mathrm{~m}$ \\
\hline Actual oil production rate $Q_{a}$ & $1056 \mathrm{t} / \mathrm{d}$ \\
\hline Eccentricity distance of horizontal well $\delta$ & $0.6 \mathrm{~m}$ \\
\hline
\end{tabular}

Therefore, this equation is seldom used in petroleum engineering because it is too small and is subject to large errors. This error is caused by the incorrect coning shape function which can not depict the actual water cresting shape.

\section{Joshi's Formula}

In 1986, based on Muskat's vertical well critical production formula, Joshi derived his horizontal well critical production formula:

$Q_{c}=q_{o v} \frac{\ln \left(r_{e} / r_{w}\right)}{\ln \left(r_{e} / r_{w}^{\prime}\right)} \cdot \frac{h^{2}-\left(h-I_{h}\right)^{2}}{h^{2}-\left(h-I_{v}\right)^{2}}$

where:

$q_{o v}=\frac{2.625 \times 10^{-3} \Delta \rho K_{h}\left[h^{2}-\left(h-I_{v}\right)^{2}\right]}{\mu B \ln \left(r_{e} / r_{w}^{\prime}\right)}$

$r_{w}^{\prime}=\frac{r_{e h}{ }^{L}}{2 a\left[1+\sqrt{1-(L / 2 a)^{2}}\right]\left(h / 2 r_{w}\right)^{h / L}}$

The following equation is obtained by combining equations (28) and (29):

$Q_{c}=\frac{2.625 \times 10^{-3} \Delta \rho K_{h}\left[h^{2}-\left(h-I_{h}\right)^{2}\right]}{\mu B_{o}\left(\ln \frac{1+\sqrt{1-(L / 2 a)^{2}}}{L / 2 a}+\frac{h}{L} \ln \frac{h}{2 r_{w}}\right)}$ 
Below is the corresponding critical production rate obtained by inputting data from Table 3 into equation (30): $Q_{c}$ $=42 \mathrm{~m}^{3} / \mathrm{d}$. Obviously, equation (30) is derived by replacing the pressure differential $(\Delta P)$ in the stationary solution equation with the critical condition $\Delta P\left[h^{2}-\left(h-I_{h}\right)^{2}\right]$, which was used by Muskat in his derivation of critical production for the vertical wells.

\section{NEW METHOD OF THE CRITICAL PRODUCTION RATE CALCULATION}

When crude oil was produced from the bottom water reservoir, in the well producing process, water moves up toward the breakthrough into the wellbore in a coning shape after production pressure difference exceeds a certain pressure. In those cases, water will break through into the oil well and oil and water are produced together. Our research purpose is to ensure the WOC does not deform in horizontal wells. In other words, how do we determine the critical production rate of horizontal wells? We also hope to know, the critical production rate in which there is no water production at any time. What is the condition without water coning? We will show one as follows:

$\Delta P=9.8 \times 10^{-3}\left(\rho_{w}-\rho_{o}\right) Z_{w}$

Equation (32) is derived on the basis of reference 6:

$Q_{c}=\frac{5.4259 \times 10^{2} K h\left[9.8 \times 10^{-3}\left(\rho_{w}-\rho_{o}\right)\right] Z_{w}}{\mu B_{o}\left(\frac{\pi r_{e}}{L}+\frac{h}{L} \ln \frac{h}{2 \pi r_{w}} \cos \frac{\pi \delta}{h}\right)}$

Re-writing equation (31), as the following:

$Q_{c}=\frac{5.4259 \times 10^{2} K h \Delta P}{\mu B_{o}\left(\frac{\pi r_{e}}{L}+\frac{h}{L} \ln \frac{h}{2 \pi r_{w}} \cos \frac{\pi \delta}{h}\right)} \times \frac{9.8 \times 10^{-3}\left(\rho_{w}-\rho_{o}\right) Z_{w}}{\Delta P}$

where:

$Q_{h}=\frac{5.4259 \times 10^{2} K h \Delta P}{\mu B_{o}\left(\frac{\pi r_{e}}{L}+\frac{h}{L} \ln \frac{h}{2 \pi r_{w}} \cos \frac{\pi \delta}{h}\right)}$

Therefore:

$J_{h}=Q_{h} / \Delta P$

Equation (32) can also be rewritten, as:

$Q_{c}=9.8 \times 10^{-3}\left(\rho_{w}-\rho_{o}\right) Z_{w} J_{h}$

The critical production is $Q_{c}=177 \mathrm{~m}^{3} / \mathrm{d}$ by inputting data from Table 3 into equation (33) or (35). The relative error of this result is $19.2 \%$ compared with Chaperon's corrected equation.

In all cases, the Chaperon, Giger and Joshi equations used to predict the critical production rate result in different results. The result of Chaperon's predicting is highest amongst these three equations. In general, for the well Tazhong 4 of Tarim oilfield in China (the well is TZ4-27-H14), if the Chaperon equation was recommended to use in the reservoirs, water will break through into well in a short time, and thus using Chaperon's equation to predict critical production rate is improper. Therefore, it is recommended to use the new method that is close to the actual data of the oilfield for critical production rate predicting.

\section{CONCLUSIONS}

1. The errors between calculation result of the Joshi equation and oilfield actual result range from $82.2 \%$ to $121.96 \%$ when Joshi's equation was used to predict horizontal well productivity in well L-3, S-1 and R-1. the errors between predicting results of corrected Joshi equation and oilfield actual results were decreased, ranging from $18.3 \%$ to $58.2 \%$, therefore, the corrected equation can also be used to predict horizontal well productivity.

2. The new productivity predicting equation is not only simple but also has high predicting accuracy when its calculation results compared with the oilfield actual results, with the largest relative error is $17.5 \%$.

3. The original derivation of Chaperon's critical production rate equation is correct, but there are errors in the simplified equation, which has been modified by the author in the paper.

4. The author suggests that the new critical production rate equation and the modified Chaperon equation both were recommended to determine the critical production rate for horizontal wells with bottom water reservoirs because its relative error is only $19.2 \%$ by comparison of critical production rate between the calculation result of new equation with the modified Chaperon's equation.

\section{NOMENCLATURE}

$K=$ Effective permeability, $\mu m^{2}$

$K_{V_{v}}=$ Vertical permeability, $\mu m^{2}$

$K_{h}=$ Horizontal permeability, $\mu m^{2}$

$\mu_{o}=$ Underground oil viscosity, $m P_{a} \bullet s$

$L \quad=$ Length of horizontal well, $m$

$r_{w} \quad=$ Radius of well bore, $m$

$r_{e h}=$ Drainage radius of horizontal well, $m$

$h \quad=$ Thickness of oil reservoir, $m$

$B_{o}=$ Oil volume factor

$r_{e} \quad=$ Drainage radius, $m$

$q^{*}=$ Dimensionless critical production rate

$\alpha=$ Middle constant

$\beta=$ Formation anisotropic coefficient, dimensionless

$\rho_{w}=$ Underground water density, $\mathrm{g} / \mathrm{cm}^{3}$

$\rho_{o} \quad=$ Underground oil density, $\mathrm{g} / \mathrm{cm}^{3}$

$\Delta \rho=$ Difference of underground water and oil density, $\mathrm{g} / \mathrm{cm}^{3}$

$Z_{s c}=$ Coning height of bottom water, $m$

$X_{A}=$ Drainage radius of vertical toward horizontal well length, $m$ 
$\delta \quad=$ Eccentricity distance of horizontal well, $m$

$Z_{w}=$ Distance from bottom of oil layer to horizontal well, $m$

$\phi \quad=$ Fluid potential

$F \quad=$ Dimensionless function

$Q_{h} \quad=$ Production well of horizontal well, $\mathrm{m}^{3} / d$

$Q_{c}=$ Critical production rate, $m^{3} / d$

$Q_{c v}=$ Critical production rate calculated by corrected Chaperon's Equation, $\mathrm{m}^{3} / d$

$I_{v} \quad=$ The distance between the oil-water contact and the top of perforated section in the vertical well, $m$

$I_{h}=$ Distance between horizontal well and the water-oil or oil- gas interface, $m$

$r_{e h}=$ Radius of drainage oil, $m$

$Q_{a}=$ Value of actual production, $\mathrm{m}^{3} / d($ tons $/$ day $=t / d)$

$\mathrm{Q}_{\mathrm{n}}=$ Calculation value from equation (17) $\mathrm{m}^{3} / \mathrm{d}$ (tons/day)

$Q_{n e}=$ Error of equation (17) versus $\mathrm{Q}_{\mathrm{a}}, \%$

$Q_{j} \quad$ Calculation value of Joshi's equation (5), $\mathrm{m}^{3} / \mathrm{d}$ (tons/day)

$Q_{j e}=$ Error of Joshi's equation (5) versus $\mathrm{Q}_{\mathrm{a}}, \%$

$Q_{j c}=$ Calculation value of equation (7), $\mathrm{m}^{3} / d$ (tons/day)

$Q_{j c e}=$ Error of equation (7) versus $\mathrm{Q}_{\mathrm{a}}, \%$

$P_{e} \quad=$ Formation pressure, $M P_{a}$

$P_{w}=$ Flow pressure in the horizontal well, $M P_{a}$.

$\Delta P=$ Producing pressure drop, $M P_{a}$

\section{APPENDIX A: POTENTIAL DIFFERENTIAL OF BOTTOM-HOLE}

According to below potential function

$\Phi=\frac{q}{4 \pi} \ln \left(\operatorname{ch} \frac{\pi X}{h}-\cos \frac{\pi\left(Z-Z_{w}\right)}{h}\right)\left(\operatorname{ch} \frac{\pi X}{h}-\cos \frac{\pi\left(Z+Z_{w}\right)}{h}\right)+c$

When $\mathrm{Z}=Z_{w}, X=r_{w}$, in terms of equation (1a), potential distribution at bottom hole was expressed as follows

$\Phi_{w}=\frac{q}{4 \pi} \ln \left(\operatorname{ch} \frac{\pi r_{w}}{h}-1\right)\left(\operatorname{ch} \frac{\pi r_{w}}{h}-\cos \frac{2 \pi Z_{w}}{h}\right)+c$

When $r_{w}<<h$, equation (2a) was simplified as follows

$\Phi_{w}=\frac{q}{2 \pi} \ln \left(\frac{\pi r_{w}}{h} \sin \frac{\pi Z_{w}}{h}\right)+c$

When $Z=Z_{w}, X=r_{e}$, and when

$\operatorname{ch} \pi X / h \gg>1 \operatorname{ch}(\pi X / h)-1 \approx \operatorname{ch}(\pi X / h)$

$\operatorname{ch}(\pi X / h)-\cos \left(2 \pi Z_{w} / h\right) \cong \operatorname{ch} \pi X / h$
Potential distribution at drainage boundary of reservoir was expressed as follows

$\Phi_{e}=\frac{q}{2 \pi} \ln \left(\operatorname{ch} \frac{\pi r_{e}}{h}\right)+c$

Therefore, potential difference at bottom hole of horizontal well

$\Delta \Phi=\Phi_{e}-\Phi_{w}=\frac{q}{2 \pi} \ln \frac{c h \pi r_{e} / h}{\frac{\pi r_{w}}{h} \sin \frac{\pi Z_{w}}{h}}+c$

When $\mathrm{r}_{\mathrm{e}}>>\mathrm{h}$

$\operatorname{ch}\left(\pi r_{e} / h\right)=(1 / 2) e^{\pi Z_{w} / h}$

Equation (5a) was further simplified, and potential differential at horizontal well bottom hole was obtained as follows:

$\Delta \Phi=\frac{q}{2 \pi}\left(\frac{\pi r_{e}}{h}+\ln \frac{h}{2 \pi r_{w}} \sin \frac{\pi Z_{w}}{h}\right)$

\section{REFERENCES}

[1] F. M. Giger, L. H. Reiss, A. P. Jourdan, "The Reservoir Engineering Aspects of Horizontal Drilling, SPE13024,', presented at SPE Annual Technical Conference and Exhibition, Houston, USA, 1984.

[2] G. L. Renard, and J. M. Duppuy, "Influence of Formation Damage on the Flow Efficiency of Horizontal wells, SPE19414," Formation Damage Control Symposium, Lafayette, Louisiana, February, 1990.

[3] S. D. Joshi, "Augmentation of Well Productivity With Slant and Horizontal Wells, SPE15375," presented at SPE Annual Technical Conference and Exhibition, New Orleans, Louisiana, 5-8 October, 1986.

[4] S. D. Joshi, “Horizontal Well Technology”. Penn Well Publishing Company; Tulsa, OK, 1991.

[5] B. J. Karcher, F. M. Giger, J. Combe, "Some Practical Formations to Predict Horizontal Well Behavior; SPE 15430," presented at SPE Annual Technical Conference and Exhibition, New Orleans, Louisiana, USA, 1986.

[6] M. Muskat, "The Flow of Homogeneous Fluids through Porous Media", International Human Resouces Development Corporation, Boston, USA, 1982, pp. 175-191.

[7] J. Bear, "Hydraulics of Groundwater, Mcgraw-Hill Series in Water Resources and Enviromental Engineering”, New York: McgrawHill Inc. 1979, 356-367.

[8] I. Chaperon, "Theoretical Study of Coning Toward Horizontal and Vertical Wells in Anisotropic Formations: Subcritical and Critical Rates, SPE15377," presented at SPE Annual Technical Conference and Exhibition, 5-8 October, New Orleans, Louisiana, USA, 1986.

[9] F. M. Giger, "Analytic 2-D Models of Water Cresting Before Breakthrough for Horizontal Wells; SPE15378," presented at SPE Annual Technical Conference and Exhibition, New Orleans, Louisiana, USA, 1986.

[10] Boyong Guo, R. L. Lee, "Determination of the Maximum WaterFree Production Rate of a Horizontal Well with Water/Oil Interface Cresting; SPE24324," presented at SPE Rocky Mountain Regional Meeting, 18-21 May, 1992, Casper, Wyoming.

[11] P. Permadi, "Practical Methods to Forecast Production of Horizontal Wells; SPE29310," presented at SPE Asia Pacific Oil and Gas Conference, Kuala Lumpur, Malaysia, 1995.

[12] B. J. Dikken, "Pressure Drop in Horizontal wells and Its Effect on Production Performance," Journal of Petroleum Technology, pp. 1426-1433, November 1990. 